Research Article

\title{
Dietary Supplementation with Inulin Modulates the Gut Microbiota and Improves Insulin Sensitivity in Prediabetes
}

\author{
Xiaojing Wang, ${ }^{1,2}$ Tong Wang, ${ }^{1}$ Qian Zhang, ${ }^{1} \mathrm{Li} \mathrm{Xu},{ }^{3}$ and Xinhua Xiao ${ }^{1}{ }^{1}$ \\ ${ }^{1}$ Department of Endocrinology, NHC Key Laboratory of Endocrinology, Peking Union Medical College Hospital, \\ Chinese Academy of Medical Sciences and Peking Union Medical College, Beijing, China \\ ${ }^{2}$ Department of Endocrinology, Beijing Tsinghua Changgung Hospital, Tsinghua University, Beijing, China \\ ${ }^{3}$ State Key Laboratory of Membrane Biology and Tsinghua-Peking Center for Life Sciences, School of Life Sciences, \\ Tsinghua University, Beijing, China
}

Correspondence should be addressed to Xinhua Xiao; xiaoxh2014@vip.163.com

Received 27 February 2021; Accepted 18 June 2021; Published 29 June 2021

Academic Editor: Anda Mihaela naciu

Copyright ( 2021 Xiaojing Wang et al. This is an open access article distributed under the Creative Commons Attribution License, which permits unrestricted use, distribution, and reproduction in any medium, provided the original work is properly cited.

Aims. Accumulating evidence indicates gut microbiota dysbiosis is involved in metabolic disorders, including prediabetes. The prebiotic inulin has been frequently reported to exert beneficial effects on the host metabolism. Here, we aimed to evaluate whether dietary supplementation with inulin modulates gut microbiota structure in prediabetes, affecting glucose and lipid metabolism. Methods. We performed a prospective single-arm study. A total of 49 subjects with prediabetes (WHO 1999 criteria) were voluntarily enrolled. Each subject received a daily supplement with $15 \mathrm{~g}$ of inulin for 6 months. Glucose and lipid metabolic parameters and gut microbiota were analyzed at baseline and at 3 and 6 months after inulin intervention. Intestinal microbiota profile was evaluated using the Illumina MiSeq platform based on V3-V4 bacterial 16S rRNA gene. Results. The mean age of 49 subjects was $56.6 \pm 6.9$ years and BMI was $25.07 \pm 3.02 \mathrm{~kg} / \mathrm{m}^{2}$. After 24 weeks of prevention, inulin significantly decreased fasting insulin $(2.38 \pm 0.50$ vs. $2.22 \pm 0.62, P=0.03)$ and 2 -hour post-OGTT insulin $(4.01 \pm 0.77$ vs. $3.74 \pm 0.76, P=0.02)$ and improved HOMA-IR $(1.05 \pm 0.53$ vs. $0.85 \pm 0.66, P=0.03)$. Gut microbiota analysis indicated that inulin supplement resulted in an increase in the relative abundance of Actinobacteria, Bifidobacteriales, Bifidobacteriaceae, Lactobacillaceae, Bifidobacterium, Lactobacillus, and Anaerostipes both at 3 and 6 months, while with a decrease in the relative abundance of Alistipes. Spearman correlation analysis revealed altered microbial community was associated with glucose and lipids metabolic parameters. Conclusions. Inulin supplementation improves insulin resistance of prediabetes and exerts beneficial effects on modulating the intestinal microbiota composition. These findings suggest that insulin may be a potentially novel and inexpensive intervention for prediabetes.

\section{Introduction}

Prediabetes represents an intermediate state between normal glucose tolerance and diabetes, which is characterized by impaired fasting glucose (IFG) and/or impaired glucose tolerance (IGT) [1]. These impairments have often been traced back to the emergence of insulin resistance, which commonly precedes the development of diabetes by several years $[2,3]$. In China, $50.1 \%$ of adults have been found to have prediabetes, and the prevalence of prediabetes was relatively high in wealthy East China village [4]. Despite prediabetes often being asymptomatic, there is a high risk of progression to type 2 diabetes in those patients [5].
Additionally, prediabetic dysglycaemia increases the risk of long-term microvascular and macrovascular complications of diabetes, such as cardiovascular disease and stroke [6, 7].

Evidence has been accumulating that gut microbiota play important roles in health maintenance. The intestinal microbiota, consisting of more than 1000 species, mediates the host metabolism by producing metabolites involved in inflammation and gut barrier integrity [8]. Gut microbial dysbiosis has been reported to be associated with metabolic disorders including type 2 diabetes, obesity, insulin resistance, and prediabetes [9]. One study showed that individuals with prediabetes have aberrant intestinal microbiota characterized by a decreased abundance of the genus 
Clostridium and the mucin-degrading bacterium A. muciniphila [10]. Therefore, microbiota-targeted interventions that help restore intestinal microbiome structure have suggested a promising strategy in the prevention of prediabetes.

In recent years, prebiotics have gained the great interest of public and researchers as they beneficially modulate gut microbiota composition [11, 12]. Inulin, a well-known prebiotic, has been frequently reported to exert a large number of beneficial effects on the host metabolism including improving glucose and lipid metabolism and protecting against weight gain in animal and human studies $[13,14]$. A RCT study recently showed that probiotics supplementation significantly increased the abundance of the Bacteroides fragilis to Escherichia coli in the adults with prediabetes [15]. However, the effect of inulin on the intestinal microbiota ecosystem in prediabetic individuals remains unclear.

Thus, in the present study, we focused on prediabetes and aimed to evaluate the effect of dietary supplementation with inulin on gut microbiota structure using a highthroughput sequencing platform. Furthermore, the association between gut microbial changes and host glucose and lipid metabolism indices were also explored.

\section{Materials and Methods}

2.1. Subjects. A total of 49 subjects were voluntarily enrolled between July 2016 and December 2017. Each participant met the following inclusion criteria: between 18 and 70 years old, BMI between 18 and $35 \mathrm{~kg} / \mathrm{m}^{2}$, fasting plasma glucose between $6.1 \mathrm{mmol} / \mathrm{L}$ and $7.0 \mathrm{mmol} / \mathrm{L}$, and $/$ or $2 \mathrm{~h}$ postprandial serum glucose levels between $7.8 \mathrm{mmol} / \mathrm{L}$ and $11.1 \mathrm{mmol} / \mathrm{L}$ following a 2-hour $75 \mathrm{~g}$ oral glucose tolerance test (the test was performed after a washout period of 4 weeks). During the washout period, subjects were asked to keep their dietary and physical activity habits. The exclusion criteria were gastrointestinal tract disease, renal and or liver disorder, psychiatric disorders, antibiotic use, probiotic, prebiotic, and symbiotic use during the past 3 months, and or during the intervention period, taking any other drugs that may affect gut microbiota and pregnancy. All participants provided written informed consent. The study was approved by the local Ethics Committee of Peking Union Medical College Hospital (No. S749), and written informed consent was obtained from all participants.

2.2. Dietary Intervention. Each subject received a daily supplement with $15 \mathrm{~g}$ of inulin for 6 months. The products were kindly supplied by Inuling Biosciences Company, Wuhan, China. The inulin powder was added in warm drinks, and compliance was evaluated by returned sachet counts. The participants were instructed not to change their dietary and physical activity habits, and they were asked to record daily diary during the intervention period to assess dietary intake, exercise, and side effects. Contact with subjects was maintained weekly during the study, and any concerns were addressed.
2.3. Biochemical Analysis and Anthropometrics. Height, weight, and blood pressure were measured at baseline and repeated at 12 weeks and 24 weeks during intervention. Liver and renal function, lipid profiles, and glycated hemoglobin (HbA1c) levels were also evaluated at each follow-up visit. Oral glucose tolerance test (OGTT) was performed at the baseline and the end of the treatment (24 weeks). HbA1c was determined using high-performance liquid chromatography (HPLC). Serum insulin and C-peptide were measured by direct chemiluminescence immunoassay (SIMENS ADVIA Centaur XP, Germany). The glucose oxidase method was used to measure the plasma glucose levels. All laboratory tests were performed after an 8-hour overnight fast. Homeostatic model assessments of islet B cell function (HOMA-B) and basal insulin resistance (HOMA-IR) were evaluated. The calculation formulas were as follows: HOMA$\mathrm{B}=$ fasting serum insulin $(\mathrm{mU} / \mathrm{L}) \times 20 /$ fasting plasma glucose in $\mathrm{mmol} / \mathrm{L}-3.5)$, HOMA-IR = fasting serum insulin $(\mathrm{mU} / \mathrm{L}) \times$ fasting plasma glucose $(\mathrm{mmol} / \mathrm{L}) / 22.5$.

2.4. Gut Microbiota Analysis. Stool samples were collected at the beginning and each follow-up visit (12 weeks and 24 weeks). All participants were provided with FloraPrep ${ }^{\mathrm{TM}}$ specimen collection kits for stool collection. Upon collection, the stool samples were immediately stored at $-80^{\circ} \mathrm{C}$. Microbial DNA was extracted from fecal samples using ZR Fecal DNA MiniPrep (D6010, Zymo Research, America) according to manufacturer's protocols. The V3-V4 hypervariable region of the $16 \mathrm{~S}$ rRNA bacterial gene was amplified by polymerase chain reaction (PCR) $\left(95^{\circ} \mathrm{C}\right.$ for $2 \mathrm{~min}$, followed by 15 cycles at $95^{\circ} \mathrm{C}$ for $30 \mathrm{~s}, 57^{\circ} \mathrm{C}$ for $30 \mathrm{~s}$, and $72^{\circ} \mathrm{C}$ for $30 \mathrm{~s}$, and a final extension at $72^{\circ} \mathrm{C}$ for $10 \mathrm{~min}$ ). Amplicons were purified using a quick PCR purification kit (Qiagen, Germany). Purified amplicons were then sequenced by Illumina MiSeq $2 * 300 \mathrm{bp}$ the paired-end sequencing platform (Suzhou Admera Med-tech Co., Ltd).

Raw data were filtered with a specific standard to obtain high-quality clean tags using QIIME (V1.8.0). The final qualified sequences were clustered as operational taxonomic units (OTUs) based on $97 \%$ similarity threshold using UPARSE. Representative sequences for each OTU were annotated with taxonomic information based on the RDP classifier version 2.2 algorithm by aligning against the GreenGene database. Alpha-diversity and beta-diversity analyses were carried out using QIIME (V1.8.0). ACE index, Shannon's index, Simpson's index, and Chaol estimates were evaluated. Permutational multivariate analysis of variance (PERMANOVA) was used to compare the betadiversity. The correlations between the relative abundance of bacterial taxa and clinical parameter were analyzed by using Spearman's correlation.

2.5. Statistical Analysis. Data were analyzed using the SPSS statistical software package version 16. Insulin, HOMA-IR, and HOMA-B were logarithmical to normal distribution and expressed as mean \pm standard deviation. A one-way repeated-measures analysis of variance with Tukey's post hoc test or paired $t$-test was used for clinical parameters 
comparisons between baseline and after intervention at each time point. $P$ value $<0.05$ was considered statistically significant.

\section{Results}

3.1. Anthropometric and Clinical Parameters. The mean age of 49 subjects was $56.6 \pm 6.9$ years (37 to 69 years old) and BMI was $25.07 \pm 3.02 \mathrm{~kg} / \mathrm{m}^{2}$. Male subjects accounted for $32.7 \%$ (16/49). After 24 weeks of prevention, inulin did not significantly modify HbA1c, 2-hour post-OGTT glycemia, and lipid profiles including total cholesterol, triglycerides, high-density lipoprotein cholesterol (HDL-c), and lowdensity lipoprotein cholesterol (LDL-c). However, inulin significantly improved fasting insulin, 2-hour post-OGTT insulin, and HOMA-IR. We also observed inulin significantly decreased fasting blood glucose at 12 weeks, but the changes were not persistent. The detailed information is given in Table 1.

3.2. Analysis of the Gut Microbiota Composition. To explore the effect of inulin on gut microbiota structure, we conducted 16s rRNA gene sequencing using fecal samples collected at weeks 0,12 , and 24 during inulin intervention. A total of 10138038 high-quality reads were obtained from 147 samples. The alpha-diversity of the gut microbiota community indicated that inulin significantly decreased the ACE richness estimates and the Shannon diversity index at the 24 weeks (Table 2). To compare the beta-diversity of intestinal microbiota before and after treatment, the PCoA analysis based on Bray-Curtis distance and unweighted UniFrac distances was carried out, and the results revealed no statistically significant differences between baseline and at each time $(P>0.05)$ (Figure 1).

However, inulin supplement significantly altered the composition of the gut microbiota. Figure 2 shows the relative abundance of intestinal microbiota at the phylum and genus level at each time point. Consistent with the previous reports, the dominant phyla were Bacteroidetes and Firmicutes in all samples. LEfSe analysis indicated that prediabetes patients were dominated with the order Desulfovibrionales, family Desulfovibrionaceae, and genus Alistipes. At 3 and 6 months after inulin supplement, the phylum Actinobacteria, the order Bifidobacteriales, family Bifidobacteriaceae and Lactobacillaceae, the genus Bifidobacterium, Lactobacillus, and Anaerostipes were enriched (Figure 3). Metastas analysis further verified the results. The abundance of Actinobacteria, Bifidobacteriales, Bifidobacteriaceae, Bifidobacterium, Lactobacillaceae, and Lactobacillus were significantly increased at 3 and 6 months compared with baseline, while Alistipes showed decrease in relative abundance (Figure 4 and Table S1). The other significantly different bacteria from the phylum level down to the genus level based on Metastas analysis are given in Table S1.

3.3. Correlation Analysis between the Fecal Microbiota and Glucose and Lipid Metabolic Parameters. To assess whether the distribution of gut microbiota and the glucose and lipid metabolic parameters were correlated, Spearman's correlation analysis was performed. Eubacterium rectale group displayed a strong positive correlation with fasting insulin, OGTT-30 min insulin, OGTT-1 h insulin, and triglyceride level. Butyricimonas and Odoribacter were positively correlated with $\mathrm{HbA1c}$ and OGTT $30 \mathrm{~min}$ glucose. Bifidobacterium showed a negative correlation with HDL-c and positive correlation with triglyceride levels. Lactobacillus exhibited a negative correlation with LDL-c, while Alistipes were negatively correlated with HDL-c (Figure 5).

\section{Discussion}

The present study explored the role of inulin on gut microbiome in prediabetes patients. The inulin's efficacy on glucose and lipid metabolism were also evaluated. Following 6 months intervention, inulin significantly ameliorated insulin resistance. This is consistent with a previous randomized crossover trial in which inulin was associated with a significant reduction in HOMA-IR in isolated-impaired fasting glucose subjects [16]. In addition, a meta-analysis showed that inulin-type carbohydrates could improve insulin resistant in T2DM, especially in obese T2DM patients [13]. Another meta-analysis included 33 RCTs which further demonstrated that inulin-type fructan (ITF) significantly reduced HOMA-IR in prediabetes or T2DM, as well as in healthy subjects [17].

Inulin, a dietary fiber, cannot be digested and absorbed by human intestine, but can be fermented by the intestinal microbiota, producing a great diversity of metabolites [18]. Inulin has been emerged as important modulators of microbial structure and increasingly investigated their potential benefits for human metabolism through alterations in the intestinal microbiota. Interestingly, the present study showed that inulin supplement significantly decreased the alpha-diversity of gut microbiota. Similarity, another previous study observed that oligofructose-enriched inulin intervention lead to a decrease in alpha-diversity in overweight adults [19]. Moreover, a recent study reported higher consumption of inulin-rich vegetables also decreased gene richness. The relationships between decreased gut microbial richness and health outcomes require further investigation.

An alteration of intestinal microbial composition by inulin in prediabetes was also observed. Inulin significantly increased the abundance of Bifidobacteriales, Bifidobacteriaceae, Bifidobacterium, Lactobacillaceae, Lactobacillus, and Anaerostipes, which were positively associated with lipid metabolic parameters. These results are supported by previous ITF intervention studies. A double-blind randomized cross-over intervention study evaluated the effect of inulin consumption on fecal microbiota composition in healthy adults with midconstipation, and specific inulin-induced changes in relative abundance of Bifidobacterium and Anaerostipes were identified [20]. Another study also indicated that dietary supplementation with inulin leaded to an increase in Bifidobacterium faecale, Anaerostipes badrus, and Actinobacteria Class [19]. In addition, a significant bifidogenic effect induced by ITF was observed in obese women [21]. Our previous study also showed that inulin 
TABLE 1: Comparison with glucose and lipid metabolism parameters before and after inulin supplementation.

\begin{tabular}{|c|c|c|c|c|c|}
\hline & M0 & M3 & M6 & $\mathrm{P} 1$ & $\mathrm{P} 2$ \\
\hline FBG (mmol/L) & $5.98 \pm 0.71$ & $5.69 \pm 0.64$ & $5.78 \pm 0.76$ & 0.01 & 0.11 \\
\hline 2-hour PBG (mmol/L) & $8.13 \pm 1.88$ & $8.22 \pm 2.19$ & $7.97 \pm 2.30$ & 1 & 1 \\
\hline HbAlc (\%) & $5.73 \pm 0.06$ & $5.76 \pm 0.56$ & $5.78 \pm 0.08$ & 1 & 0.63 \\
\hline $\mathrm{TG}(\mathrm{mmol} / \mathrm{L})$ & $1.37 \pm 0.10$ & $1.29 \pm 0.08$ & $1.29 \pm 0.11$ & 0.62 & 0.37 \\
\hline $\mathrm{HDL}-\mathrm{c}(\mathrm{mmol} / \mathrm{L})$ & $1.38 \pm 0.04$ & $1.41 \pm 0.05$ & $1.37 \pm 0.05$ & 1 & 1 \\
\hline $\mathrm{TC}(\mathrm{mmol} / \mathrm{L})$ & $4.93 \pm 1.06$ & $4.78 \pm 0.98$ & $4.69 \pm 0.99$ & 0.62 & 0.37 \\
\hline $\mathrm{LDL}-\mathrm{c}(\mathrm{mmol} / \mathrm{L})$ & $3.08 \pm 0.10$ & $3.03 \pm 0.13$ & $3.09 \pm 0.85$ & 1 & 1 \\
\hline FINS (mU/L) & $2.38 \pm 0.50$ & 一 & $2.22 \pm 0.62$ & 一 & 0.03 \\
\hline PINS (mU/L) & $4.01 \pm 0.77$ & - & $3.74 \pm 0.76$ & - & 0.02 \\
\hline HOMA-IR & $1.05 \pm 0.53$ & - & $0.85 \pm 0.66$ & - & 0.03 \\
\hline HOMA-B & $4.51 \pm 0.58$ & - & $4.46 \pm 0.66$ & - & 0.37 \\
\hline
\end{tabular}

Data are presented as mean \pm SD. FBG, fasting blood glucose; $2 \mathrm{PBG}$, 2-hour postload plasma glucose; HbAlc, glycated hemoglobin; TG, triacylglycerol; HDLc, high-density lipoproteins; TC, total cholesterol; LDL-c, low-density lipoproteins; FINS, fasting plasma insulin; PINS, 2-hour postload plasma insulin. P1: M0 vs. M3; P2: M0 vs. M6. $P<0.05$ is highlighted in bold. M0, baseline; M3, at three months after inulin intervention; M6, at six months after inulin intervention.

TABLE 2: Alpha-diversity before and after inulin supplementation.

\begin{tabular}{lcccc}
\hline & M0 & M3 & M6 & P1 \\
\hline ACE & $238.61 \pm 66.10$ & $224.12 \pm 58.49$ & $220.59 \pm 60.21$ & 0.20 \\
Chao1 & $243.13 \pm 67.43$ & $227.69 \pm 60.65$ & $227.05 \pm 58.49$ & 0.16 \\
Shannon & $4.89 \pm 0.80$ & $4.64 \pm 0.77$ & $4.67 \pm 0.69$ & 0.04 \\
Simpson & $0.92 \pm 0.05$ & $0.90 \pm 0.05$ & $0.91 \pm 0.05$ & 0.10 \\
\hline
\end{tabular}

Data are presented as mean \pm SD. P1 : M0 vs. M3; P2:M0 vs. M6. $P<0.05$ is highlighted in bold. M0, baseline; M3, at three months after inulin intervention; M6, at six months after inulin intervention.

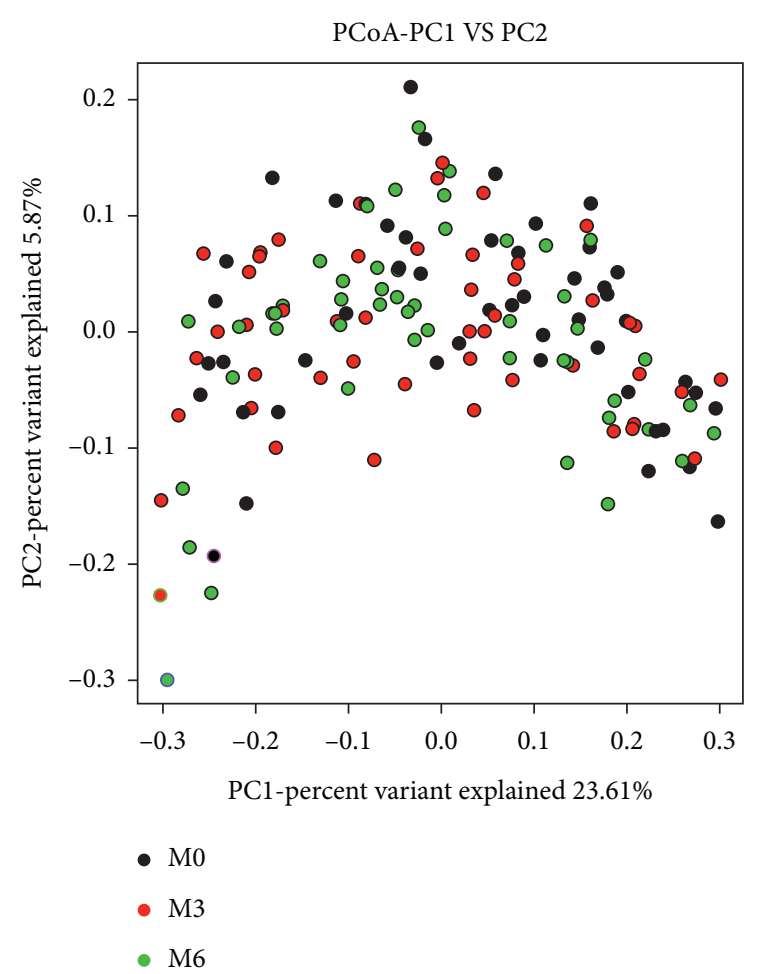

(a)

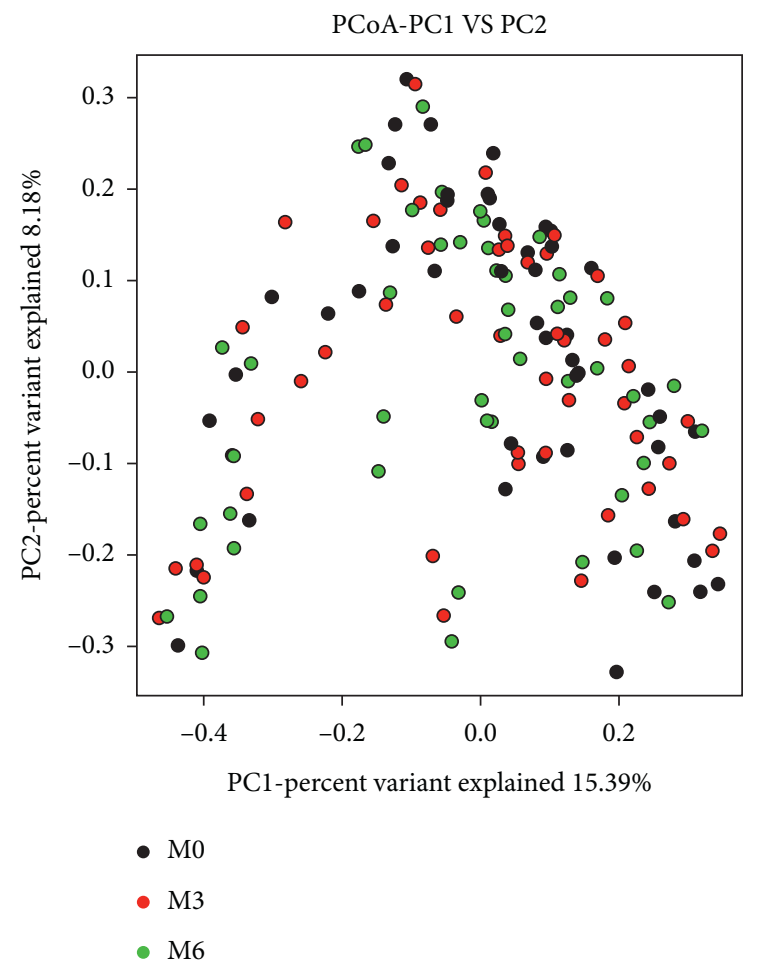

(b)

Figure 1: Principal component analysis plot based on Bray-Curtis distance (a) and unweighted UniFrac distances (b) for fecal microbiota. M0, baseline; M3, at three months after inulin intervention; M6, at six months after inulin intervention. 


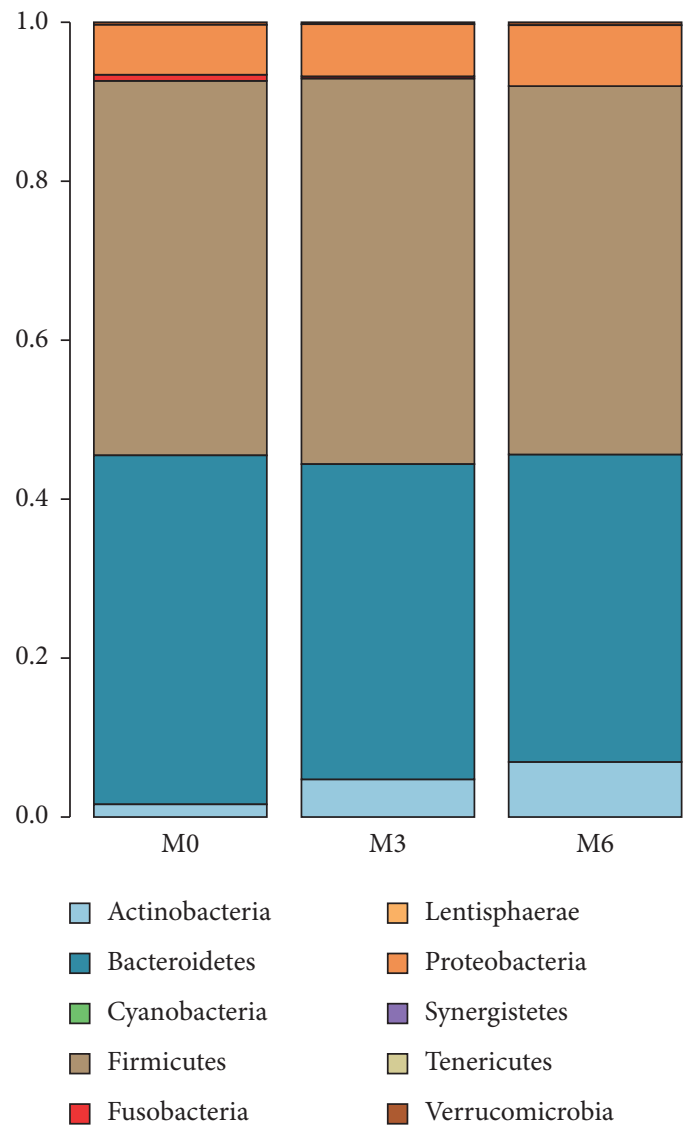

(a)

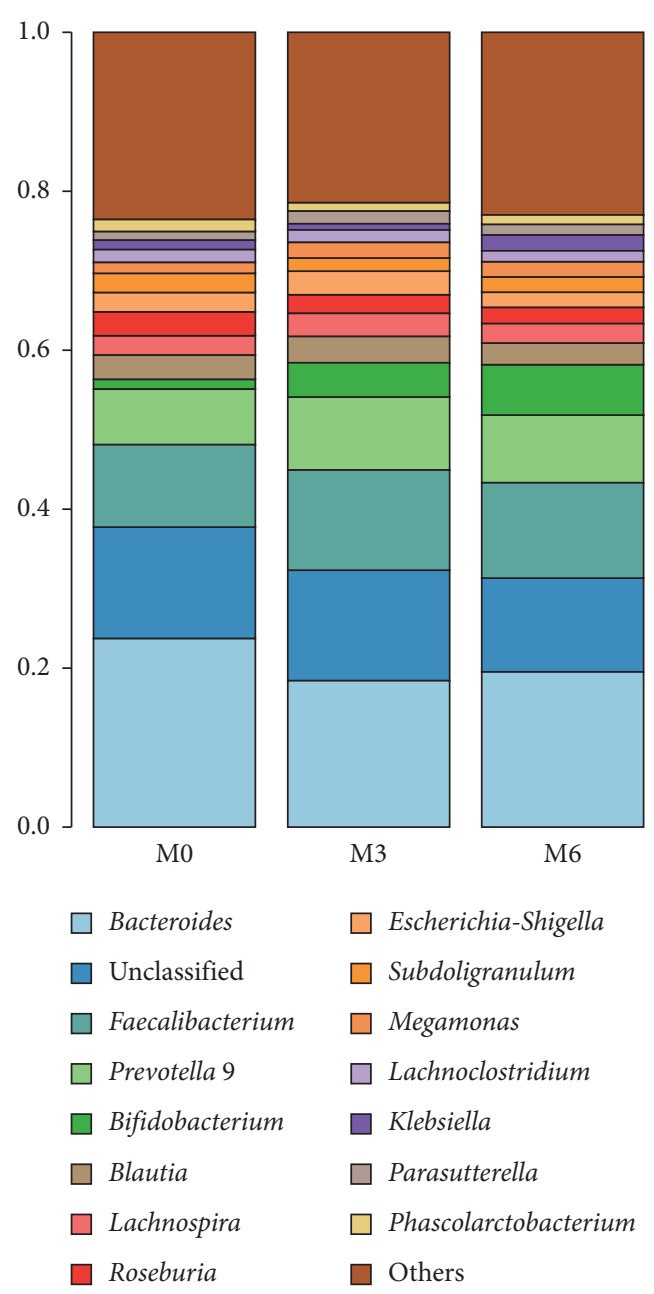

(b)

Figure 2: The relative abundance of bacteria at the phylum level (a) and genus level (b) before and after inulin supplement. M0, baseline; M3, at three months after inulin intervention; M6, at six months after inulin intervention.

supplement increased the relative abundance of Bifidobacterium_breve in offspring from high-fat diet- (HFD-) fed dams [22].

Bifidobacterium and Lactobacillus had been reported to be poorly represented in the fecal samples of diabetes patients and positively impact insulin sensitivity $[23,24]$. The two genera were well-known short chain fatty acids (SCFAs) producing bacterium [16]. SCFAs have been shown to stimulate the secretion of glucagon-like peptide-1 (GLP-1) and peptide YY by binding to the G-couple receptors on intestinal L cells and to promote energy expenditure. Additionally, SCFAs have been shown to improve systemic inflammation via reducing intestinal permeability and endotoxaemia, demonstrated to impair insulin signaling and insulin sensitivity [25]. Both animal and human studies have revealed an improved gut barrier function by increasing Bifidobacterium abundance $[21,26]$. Thus, the beneficial effect of inulin on insulin sensitivity may be partly attributed to the enhanced abundance of SCFAs producing genera.

The present study indicated that inulin supplementation induced a reduction in the abundance of Alistipes. Alistipes have been shown to be highly abundant in T2DM patients and positively correlate with the inflammatory state, driving insulin resistance $[27,28]$. A previous study found that probiotics decreased the abundance of Alistipes in mice [29]. HFD intervention was associated with increased Alistipes [30]. The sulfate-reducing bacteria of the family Desulfovibrionaceae are key producers of endotoxins. The relative abundance of Desulfovibrionaceae has been reported to be positively associated with plasma lipopolysaccharide levels, which had potent inflammation-inducing capacity and damage gut barrier [31]. Proinflammatory cytokine production was involved in insulin resistance-related metabolic disease. Several studies had demonstrated a marked increase in the abundance of intestinal Desulfovibrionaceae both in HFD-induced obesity mice and obesity human subjects $[32,33]$. Moreover, Desulfovibrionaceae were also enhanced in mice with impaired glucose tolerance [34]. In agreement with these results, our findings showed that the family Desulfovibrionaceae dominated in prediabetes before inulin intervention, but not found at 3 and 6 months after inulin supplement. Taken together, the inhibition of potential 


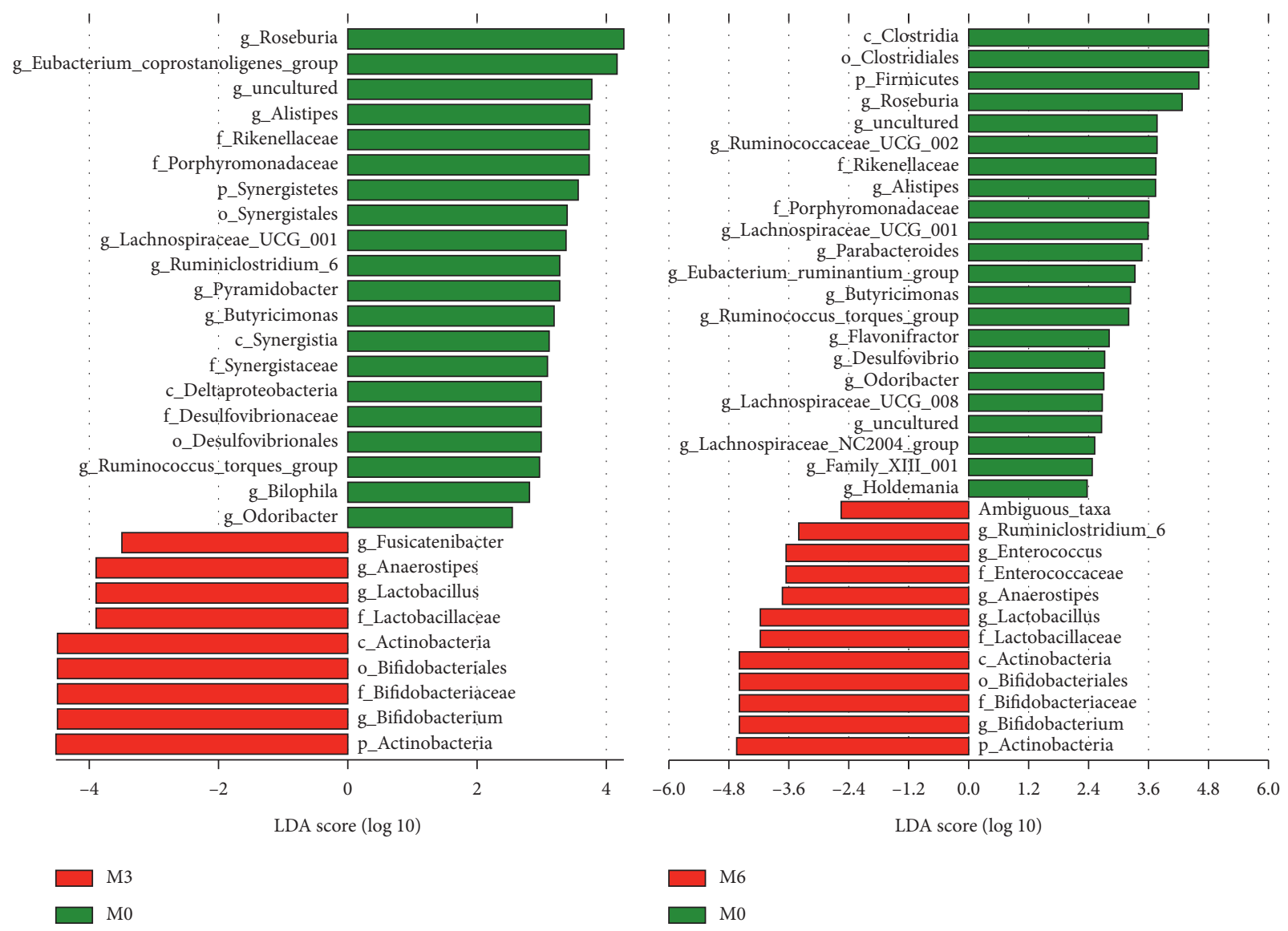

(a)

(b)

Figure 3: The LEfSe analysis of the gut microbiota from the phylum level down to the genus level. (a) M0 vs. M3; (b) M0 vs. M6. M0, baseline; M3, at three months after inulin intervention; M6, at six months after inulin intervention.

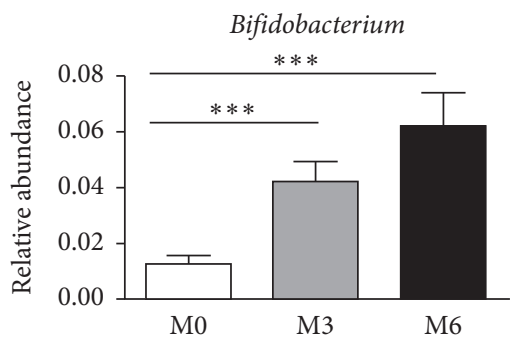

(a)

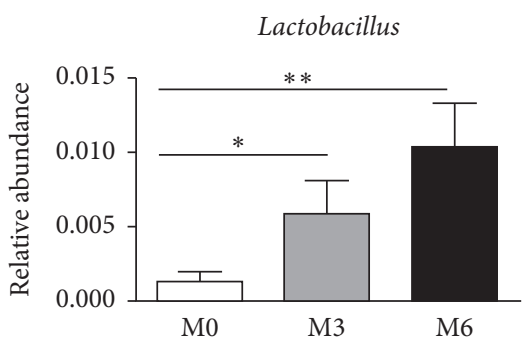

(b)

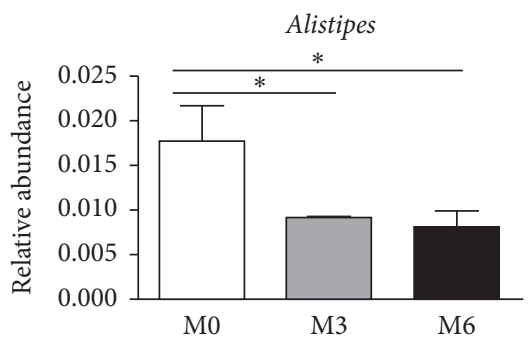

(c)

Figure 4: The relative abundance of bacterial taxa at the genus level before and after before and after inulin supplement. (a) Bifidobacterium; (b) Lactobacillus; (c) Alistipes M0, baseline; M3, at three months after inulin intervention; M6, at six months after inulin intervention.

pathogen-like bacteria and increase in SCFAs producing beneficial bacterium might be responsible alleviating insulin resistance.

There were several limitations in this study. First, the sample size was relatively small. Second, this study was a single-arm, open-label, prepost intervention trial. Lack of an independent control group was another limitation, and potential bias may exist. Third, we were unable to determine the causal relationship between improvements in insulin sensitivity and changes in gut microbiota composition promoted by inulin. The underlying molecular mechanisms warrant further exploration.

In conclusion, the present study indicates that inulin supplementation significantly improved insulin resistance in prediabetes. Our data also showed that inulin was a unique gut microbiota modulating agent that enriched the beneficial bacteria including Bifidobacterium and Lactobacillus and inhibited the potential pathogen-like bacteria, 


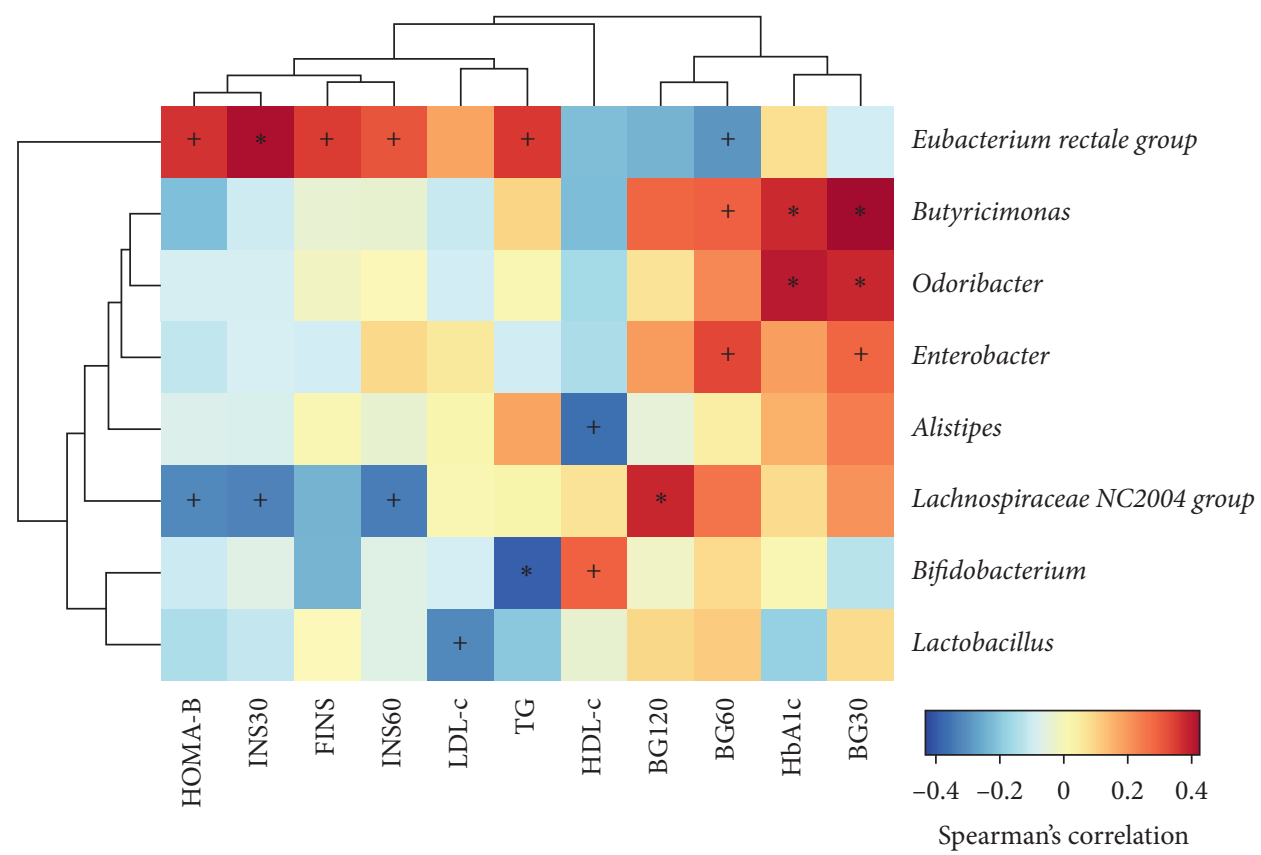

FiguRE 5: Heat map showing spearman correlations between different gut microbiota at the genus level and glucose and lipid metabolic parameters. ${ }^{*} P<0.05,+q<0.05$.

such as Alistipes and Desulfovibrionaceae. The inulin-induced metabolic response is possibly associated with the changes of gut microbiota composition. These findings suggest that inulin may be a potentially novel and inexpensive intervention for prediabetes. However, owing to the small sample size and absence of an independent control group of this study, larger scale and randomized controlled trials are therefore warranted to further confirm our findings.

\section{Data Availability}

The data used to support the findings of this study are available from the corresponding author upon request.

\section{Consent}

Informed consent was obtained from all subjects included in the study.

\section{Conflicts of Interest}

The authors declare that they have no conflicts of interest.

\section{Acknowledgments}

The work was supported by the National Key R\&D Program of China (2017YFC1309603 and 2018YFC2001100), National Natural Science Foundation of China (81170736, 81570715, 81870579, and 82000746), and Beijing Natural Science Foundation (7202163 and 7214275). The authors thank for Inuling Biosciences Company (Wuhan, China) supplying the inulin products and all study participants in this study.

\section{Supplementary Materials}

Supplementary Table S1. The significantly different bacteria from the phylum level down to the genus level based on Metastas analysis. (Supplementary Materials)

\section{References}

[1] American Diabetes Association, "Classification and diagnosis of diabetes: standards of medical care in diabetes-2018," Diabetes Care, vol. 41, no. Suppl 1, pp. S13-S27, 2018.

[2] B. Brannick, A. Wynn, and S. Dagogo-Jack, "Prediabetes as a toxic environment for the initiation of microvascular and macrovascular complications," Experimental Biology and Medicine, vol. 241, no. 12, pp. 1323-1331, 2016.

[3] S. Dagogo-Jack, H. Askari, and G. Tykodi, "Glucoregulatory physiology in subjects with low-normal, high-normal, or impaired fasting glucose," The Journal of Clinical Endocrinology \& Metabolism, vol. 94, no. 6, pp. 2031-2036, 2009.

[4] X. Yu, F. Duan, D. Lin et al., "Prevalence of diabetes, prediabetes, and associated factors in an adult Chinese population: baseline of a prediabetes cohort study," International Journal of Endocrinology, vol. 2020, Article ID 8892176, 8 pages, 2020.

[5] V. A. Salunkhe, R. Veluthakal, S. E. Kahn, and D. C. Thurmond, "Novel approaches to restore beta cell function in prediabetes and type 2 diabetes," Diabetologia, vol. 61, no. 9, pp. 1895-1901, 2018.

[6] Y. Huang, X. Cai, W. Mai, M. Li, and Y. Hu, "Association between prediabetes and risk of cardiovascular disease and all cause mortality: systematic review and meta-analysis," British Medical Journal, vol. 355, p. i5953, 2016.

[7] A. Sinha, H. Ning, F. S. Ahmad et al., "Association of fasting glucose with lifetime risk of incident heart failure: the lifetime risk pooling project," Cardiovascular Diabetology, vol. 20, no. 1, p. 66, 2021. 
[8] J. A. Gilbert, M. J. Blaser, J. G. Caporaso, J. K. Jansson, S. V. Lynch, and R. Knight, "Current understanding of the human microbiome," Nature Medicine, vol. 24, no. 4, pp. 392-400, 2018.

[9] E. E. Canfora, R. C. R. Meex, K. Venema, and E. E. Blaak, "Gut microbial metabolites in obesity, NAFLD and T2DM," Nature Reviews Endocrinology, vol. 15, no. 5, pp. 261-273, 2019.

[10] K. H. Allin, V. Tremaroli, V. Tremaroli et al., "Aberrant intestinal microbiota in individuals with prediabetes," Diabetologia, vol. 61, no. 4, pp. 810-820, 2018.

[11] G. R. Gibson and M. B. Roberfroid, "Dietary modulation of the human colonic microbiota: introducing the concept of prebiotics," The Journal of Nutrition, vol. 125, no. 6, pp. 1401-1412, 1995.

[12] N. M. Delzenne, A. M. Neyrinck, and P. D. Cani, "Modulation of the gut microbiota by nutrients with prebiotic properties: consequences for host health in the context of obesity and metabolic syndrome," Microbial Cell Factories, vol. 10, no. Suppl 1, p. S10, 2011.

[13] F. Liu, M. Prabhakar, J. Ju, H. Long, and H.-W. Zhou, "Effect of inulin-type fructans on blood lipid profile and glucose level: a systematic review and meta-analysis of randomized controlled trials," European Journal of Clinical Nutrition, vol. 71, no. 1, pp. 9-20, 2017.

[14] M. Roberfroid, G. R. Gibson, L. Hoyles et al., "Prebiotic effects: metabolic and health benefits," British Journal of Nutrition, vol. 104, no. Suppl 2, pp. S1-S63, 2010.

[15] N. Kassaian, A. Feizi, S. Rostami, A. Aminorroaya, M. Yaran, and M. Amini, "The effects of 6 mo of supplementation with probiotics and synbiotics on gut microbiota in the adults with prediabetes: a double blind randomized clinical trial," $\mathrm{Nu}$ trition, vol. 79-80, Article ID 110854, 2020.

[16] N. Kassaian, A. Feizi, A. Aminorroaya, and M. Amini, "Probiotic and synbiotic supplementation could improve metabolic syndrome in prediabetic adults: a randomized controlled trial," Diabetes \& Metabolic Syndrome: Clinical Research \& Reviews, vol. 13, no. 5, pp. 2991-2996, 2019.

[17] L. Wang, H. Yang, H. Huang et al., "Inulin-type fructans supplementation improves glycemic control for the prediabetes and type 2 diabetes populations: results from a GRADEassessed systematic review and dose-response meta-analysis of 33 randomized controlled trials," Journal of Translational Medicine, vol. 17, no. 1, p. 410, 2019.

[18] G. R. Gibson, H. M. Probert, J. V. Loo, R. A. Rastall, and M. B. Roberfroid, "Dietary modulation of the human colonic microbiota: updating the concept of prebiotics," Nutrition Research Reviews, vol. 17, no. 2, pp. 259-275, 2004.

[19] E. S. Chambers, C. S. Byrne, D. J. Morrison et al., "Dietary supplementation with inulin-propionate ester or inulin improves insulin sensitivity in adults with overweight and obesity with distinct effects on the gut microbiota, plasma metabolome and systemic inflammatory responses: a randomised cross-over trial," Gut, vol. 68, no. 8, pp. 1430-1438, 2019.

[20] D. Vandeputte, G. Falony, S. Vieira-Silva et al., "Prebiotic inulin-type fructans induce specific changes in the human gut microbiota," Gut, vol. 66, no. 11, pp. 1968-1974, 2017.

[21] E. M. Dewulf, P. D. Cani, S. P. Claus et al., "Insight into the prebiotic concept: lessons from an exploratory, double blind intervention study with inulin-type fructans in obese women," Gut, vol. 62, no. 8, pp. 1112-1121, 2013.

[22] Q. Zhang, X. Xiao, J. Zheng et al., "Influence of maternal inulin-type prebiotic intervention on glucose metabolism and gut microbiota in the offspring of C57BL mice," Frontiers in Endocrinology, vol. 10, p. 675, 2019.

[23] A. S. Andreasen, N. Larsen, T. Pedersen-Skovsgaard et al., "Effects ofLactobacillus acidophilusNCFM on insulin sensitivity and the systemic inflammatory response in human subjects," British Journal of Nutrition, vol. 104, no. 12, pp. 1831-1838, 2010.

[24] X. Wu, C. Ma, L. Han et al., "Molecular characterisation of the faecal microbiota in patients with type II diabetes," Current Microbiology, vol. 61, no. 1, pp. 69-78, 2010.

[25] Y. A. Kim, J. B. Keogh, and P. M. Clifton, "Probiotics, prebiotics, synbiotics and insulin sensitivity," Nutrition Research Reviews, vol. 31, no. 1, pp. 35-51, 2018.

[26] P. D. Cani, A. M. Neyrinck, F. Fava et al., "Selective increases of bifidobacteria in gut microflora improve high-fat-dietinduced diabetes in mice through a mechanism associated with endotoxaemia," Diabetologia, vol. 50, no. 11, pp. 2374-2383, 2007.

[27] J. Qin, Y. Li, Z. Cai et al., "A metagenome-wide association study of gut microbiota in type 2 diabetes," Nature, vol. 490, no. 7418, pp. 55-60, 2012.

[28] L. Zhu, L. Sha, K. Li et al., "Dietary flaxseed oil rich in omega-3 suppresses severity of type 2 diabetes mellitus via anti-inflammation and modulating gut microbiota in rats," Lipids in Health and Disease, vol. 19, no. 1, p. 20, 2020.

[29] Y.-N. Wang, X.-C. Meng, Y.-F. Dong et al., "Effects of probiotics and prebiotics on intestinal microbiota in mice with acute colitis based on 16S rRNA gene sequencing," Chinese Medical Journal, vol. 132, no. 15, pp. 1833-1842, 2019.

[30] Y. Wan, F. Wang, J. Yuan et al., "Effects of dietary fat on gut microbiota and faecal metabolites, and their relationship with cardiometabolic risk factors: a 6-month randomised controlled-feeding trial," Gut, vol. 68, no. 8, pp. 1417-1429, 2019.

[31] L. Weglarz, Z. Dzierzewicz, B. Skop et al., "Desulfovibrio desulfuricans lipopolysaccharides induce endothelial cell IL-6 and IL-8 secretion and E-selectin and VCAM-1 expression," Cellular \& Molecular Biology Letters, vol. 8, no. 4, pp. 9911003, 2003.

[32] S. Xiao, N. Fei, X. Pang et al., "A gut microbiota-targeted dietary intervention for amelioration of chronic inflammation underlying metabolic syndrome," FEMS Microbiology Ecology, vol. 87, no. 2, pp. 357-367, 2014.

[33] M. A. Hildebrandt, C. Hoffmann, S. A. Sherrill-Mix et al., "High-fat diet determines the composition of the murine gut microbiome independently of obesity," Gastroenterology, vol. 137, no. 5, pp. 1716-1724, 2009.

[34] C. Zhang, M. Zhang, S. Wang et al., "Interactions between gut microbiota, host genetics and diet relevant to development of metabolic syndromes in mice," The ISME Journal, vol. 4, no. 2, pp. 232-241, 2010. 